\title{
Alternativas a la pena Privativa de la Libertad
}

\section{Farid Samir Benavides Vanegas*}

Recibido: 16 octubre 2016

\section{Resumen}

La pena privativa de la libertad ha entrado en crisis en América Latina, en general, y en Colombia, en particular. El aumento del número de personas privadas de la libertad hace que los presupuestos nacionales sean cada vez más altos, una situación que a largo plazo será insostenible. Es preciso buscar alternativas a la pena privativa de la libertad, pero que no aumenten la red de control y que no supongan un costo ni un control mayor que el de la pena de prisión. En este texto no me ocupo de analizar la crisis de la prisión en América Latina y Europa, sino de las alternativas que se plantean en la literatura para hacerle frente. Se trata de una simple revisión de las diversas opciones. En otro texto más amplio me ocupo de la cuestión en su totalidad.

Palabras clave: Alternativa a prisión, Pena privativa, libertad, Reparación del daño, Derechos de víctimas, Rehabilitar.

\section{Alternatives to the privative penalty of freedom}

\begin{abstract}
The penalty of deprivation of liberty has entered into crisis in Latin America, in general, and in Colombia, in particular. The increase in the number of persons deprived of their liberty makes national budgets higher and higher, a situation which in the long run will be unsustainable. It is necessary to look for alternatives to the custodial sentence, but not to increase the control network and not to impose a cost or control greater than the penalty of imprisonment. In this text I am not concerned with analyzing the prison crisis in Latin America and Europe, but with the alternatives that are presented in the literature to deal with it. It is a simple review of the various options. In another larger text I deal with the question in its entirety.
\end{abstract}

\footnotetext{
*Fs.benavides@uniandes.edu.co Universidad de los Andes, Colombia.
} 
Key words: Alternative to prison, Privatization, Freedom, Harm repair, Victims rights, Rehabilitation.

\section{Sumario:}

I. Introducción, II. Alternativas a la Prisión, III. Sanciones en la Comunidad, IV. Justicia Restaurativa, Fuentes de consulta

\section{Introducción}

En los últimos años presenciamos una crisis de la pena privativa de la libertad a nivel global. Esta crisis se da por un exceso en el uso de la privación de la libertad, produciendo un aumento de personas en los establecimientos de reclusión y, como consecuencia del retraso en la construcción de infraestructura y sus altos costos, un hacinamiento cada vez más preocupante. Pero también se da por una falta de fe en el uso de la pena privativa de la libertad, lo que ha llevado a que en países como Holanda y Suecia se estén cerrando cárceles por falta de internos que ameriten tenerlas abiertas. Lamentablemente en América Latina, en general, nos encontramos con la primera de las crisis y no con la segunda, al punto que se habla del renacimiento de la prisión en la región.

La pena privativa de la libertad ha entrado en crisis en América Latina, en general, y en Colombia, en particular. El aumento del número de personas privadas de la libertad hace que los presupuestos nacionales sean cada vez más altos, una situación que a largo plazo será insostenible. Es preciso buscar alternativas a la pena privativa de la libertad, pero que no aumenten la red de control y que no supongan un costo ni un control mayor que el de la pena de prisión. En este texto no me ocupo de analizar la crisis de la prisión en América Latina y Europa, sino de las alternativas que se plantean en la literatura para hacerle frente. Se trata de una simple revisión de las diversas opciones. En otro texto más amplio me ocupo de la cuestión en su totalidad. 


\section{Alternativas a la Prisión}

Como consecuencia de la crisis de la pena privativa de la libertad, se han desarrollado diversas experiencias de alternativas a la prisión. Desde mecanismos que limitan la detención preventiva hasta medidas que buscan evitar que la persona cumpla la sanción penal privada de la libertad, las medidas todas han girado alrededor de la idea de alternativas a la prisión, esto es, medidas en donde la pena privativa de la libertad conserva su centralidad y solo se buscan alternativas a su uso. Pero medidas que tengan valor en sí mismas - con la salvedad de la multa han tenido poco desarrollo.

La búsqueda de fórmulas alternativas a la prisión tiene una larga historia y ha sido dividida en diferentes eras. Esto no quiere decir que esta sea una historia de progreso, sino que en cada momento ha imperado una forma diferente de ver el uso de la pena privativa de la libertad. Hasta comienzos de la década del setenta, la única medida disponible era la orden de libertad controlada (probation) y era considerada como una medida alternativa a ser impuesta en el proceso de determinación de la sentencia. Bottoms, Rex y Robinson dividen las eras de la siguiente manera: ${ }^{1}$

- La era del penal welfarism: La idea central que caracteriza a este periodo consistía en que las personas que cometían delitos lo hacían por alguna carencia de tipo personal, social o educativa. La sanción entonces tiene la finalidad de dar cuenta de las necesidades de tratamiento de la persona y de ayudarla a su reintegración en la sociedad mediante programas de reeducación, de rehabilitación, de resocialización, etc. El declive del ideal rehabilitador y la idea de que nada funcionaba hizo que se abandonaran los ideales RE dentro de la ejecución de la pena. En algunos países como los Estados Unidos se optó por un regreso a la idea justicia (just deserts) y por tanto por una concepción más retributiva de la pena.

1 Anthony Bottoms, Sue Rex \& Gwen Robinson. Alternatives to Prison. Options for an insecure society. Portland: Willan Publishing, 2004. Pp. 2. 
- La era de las alternativas a la custodia: Frente a la crisis del modelo rehabilitador, se comenzaron a buscar opciones que evitaran el daño y los costos de la privación de la libertad. En la Criminal Justice Act de 1967 en los Estados Unidos se introdujeron formulas de condenas más cortas y de suspensión de su ejecución si se cumplían ciertas condiciones. En 1972 se introdujo la orden de trabajo en servicio de la comunidad como parte de las alternativas a la privación de la libertad. La misión principal de estas alternativas era lograr la reducción del número de personas privadas de la libertad. Sin embargo, no solo no lo logró, sino que el hacinamiento siguió aumentando. La investigación empírica mostró que solo en la mitad de los casos se sustituyeron las penas privativas de la libertad por modelos no privativos. Su uso trajo como consecuencia negativa la ampliación de la red de control y el aumento de la severidad, esto es, personas que en otras circunstancias no habrían sido objeto del sistema penal lo fueron por la existencia de la alternativa y sanciones que en otro tiempo fueron leves, se endurecieron para evitar que la persona gozara de la alternativa a la prisión. ${ }^{2}$

- La era de sanciones en la comunidad: El modelo de justicia y su idea del just deserts fue aplicado en relación con las medidas no privativas de la libertad. Teniendo en cuenta que el objetivo principal de la pena es reconocer una culpa y producir la vergüenza del infractor, las penas deben reflejarlo para que se considere que son proporcionales a la conducta realizada. La Criminal Justice Act de 1991 estableció una modalidad de imposición de sentencia basada en la cuestión del merito. Esta ley acuñó el término sentencia en comunidad para cubrir las sanciones intermedias entre la privación de la libertad y la pena de multa. Se trataba de justificar sanciones para conductas suficientemente serias como para requerir una cierta intromisión y no tan serias como para ameritar la privación de la libertad.

2 Un efecto similar es el que se causó en Colombia con la introducción del sistema oral acusatorio y el principio de libertad. Para controlar sus efectos se aprobó la ley 890 de 2005 que produjo un incremento del número de personas en prisión. 
- La era de la nueva generación de penas en comunidad: Esta era se caracteriza por varias modalidades de aplicación de penas. Entre ellas están las siguientes:

- Penas en la comunidad. Esto busca tranquilizar a las comunidades sobre la relación entre medidas no privativas de la libertad y la seguridad de la ciudadanía.

- Tecnología: Se ha aumentado el uso de la tecnología y hay cada vez mayor dependencia de su uso. Las cámaras de vigilancia para prevenir la comisión de delitos van acompañadas del uso de brazaletes electrónicos para evitar la evasión del infractor o de doble vía para evitar que éste se acerque a sus víctimas, en especial en casos de violencia doméstica.

- Mejora en la administración de los programas y creación de asociaciones entre las diferentes agencias, de manera que el infractor no es visto de manera unidimensional sino en todas sus necesidades. Como se verá más adelante, por ejemplo, los liaison de los Tribunales de Tratamiento de Drogas en Canadá sirven como punto nodal de las diferentes agencias de prestación de servicios sociales. Pues se reconoce que una vivienda adecuada, servicios médicos, de educación, de protección social son esenciales para evitar que las personas recaigan en una situación de vulnerabilidad que les lleve a cometer nuevos delitos.

Esta era también se caracteriza por los siguientes elementos:

- Evaluación empírica de los diferentes programas, de manera que se atiende al logro de sus objetivos y no a sus bases ideológicas.

- Creatividad y mezcla de programas. La búsqueda de aquello que funciona en materia de prevención del delito ha llevado a que se tengan 
en cuenta programas variados que logren el objetivo de la inclusión social de los infractores de la ley penal.

- Reparaciones y los derechos de las víctimas. Una intención restaurativa acompaña a todas estas medidas. Esto es, no basta simplemente con que se evite el uso de la pena privativa de la libertad, sino que es necesario que se tengan en cuenta las necesidades de la víctima y el impacto que el delito ha producido en sus vidas.

Es importante tener en cuenta cuáles son los objetivos de las alternativas desarrolladas, pues no es lo mismo un sistema que se desarrolla con el fin de reducir el número de personas en la cárcel que uno que busca reducir el dolor causado por el sistema penal.

Así, si una alternativa busca reducir el número de personas privadas de la libertad, el principal indicador para medir su éxito o fracaso es cuantas personas salen de la cárcel o evitan su ingreso a ella como consecuencia del empleo del mecanismo. De la misma manera, si un mecanismo tiene como su principal finalidad reducir los efectos negativos del sistema, pero no incidir de manera significativa en el número de personas en la cárcel, el indicador debe ser precisamente cuánto ha influido en la reducción de los efectos negativos para las personas que se han beneficiado de él, y no se le puede juzgar por su poco impacto en el número de personas en prisión. Confundir estos objetivos e indicadores puede conducir a una valoración deficiente de alternativas que son útiles para unos propósitos pero no para otros.

Igualmente, es importante conocer las causas del aumento de la presencia de personas en la prisión, pues existe la tendencia a asociar el aumento en el número de internos/as con el aumento de la criminalidad. Sin embargo, lo que muestran los estudios empíricos sobre la materia es que no existe una relación directa entre el aumento de personas en la cárcel con el aumento de la criminalidad. Todo lo contrario. A una mayor reducción de la tasa de criminalidad se ha dado un mayor número de personas en prisión. ¿AA qué se debe que haya menos crimen y más 
presos? $?^{3}$ Una respuesta posible está dada por una política criminal que hace de la prisión su instrumento principal.

Un aspecto que se destaca en la literatura es que la pena privativa de la libertad contribuye a hacer más gravosa la situación de exclusión de una persona. Por ello desde diversos campos en la literatura, y superando el debate de los penalistas entre las finalidades retributivas y preventivas de la pena, se habla de la sanción penal con un fin comunicativo. Para el filósofo inglés Anthony Duff, la pena "debería ser un proceso comunicativo entre el infractor y la comunidad política: busca comunicarle a aquél la censura que su crimen merece; llevarlo a que reconozca y se arrepienta del delito como algo malo que debe reparar moralmente; llevarlo a que haga esa reparación sometiéndose a una penalidad que le imponga cargas y que constituya y comunique una disculpa a aquellos a quienes le ha causado daño; y de ese modo se reconcilie con la comunidad de la que se ha burlado. La pena es también una empresa reformadora: el infractor que se arrepiente del crimen es llevado a reconocer la necesidad de reformar su conducta futura; y su pena debería ayudarle, cuando sea apropiado, en este proceso, como lo hace por ejemplo el sistema de libertad bajo control". ${ }^{4}$ Por ello las alternativas deben tener en cuenta que no se trata de repetir los problemas de la privación de la libertad, sino de limitar sus efectos negativos, dentro de los cuales se encuentra la exclusión social.

\section{Sanciones en la Comunidad}

Como lo muestra una investigación hecha en el Reino Unido sobre las sanciones alternativas en 1977 y en 2005, las sanciones alternativas a la prisión suelen ser vistas como la opción más suave, en tanto la prisión suele ser vista como la opción más dura. Esto es, cuando se habla de sanciones comunitarias se suele afirmar que los gobiernos se han vuelto suaves en su tratamiento del delito (soft

3 Mark Kleiman. When brute force fails. How to have less crime and less punishment. Princeton: Princeton University Press, 2009.

4 R.A. Duff. "Penance, punishment, and the limits of the community". En Michael Tonry. Why punish? How much? A reader on punishment. Oxford: Oxford University Press, 2011. Pp. 179. 
on crime), dejando de lado el hecho que no todos los infractores de la ley penal van a la prisión y, sobre todo, que el control ejercido por ciertas medidas no es menor o más suave que el de la prisión, solo que se hace en libertad. ${ }^{5}$

La opinión pública asocia la imposición de una pena con la imposición de una sanción que suponga privación de la libertad. A esta discusión se le ha asociado la idea de la comunidad, y se ha hablado de sanción en la comunidad -no por la comunidad-; de rehabilitación comunitaria; y de sanciones inteligentes (smart sentencing). Incluso se ha llegado a hablar de que es como la bomba de neutrón, que deja al infractor intacto, pero que acaba con su comportamiento criminal (Worral \& Hoy, 2005: xvi). Estos programas se caracterizan por estar más orientados hacia el bienestar del infractor que a su exclusión, pero a la vez se caracterizan por una supervisión intensa, por programas de rehabilitación estrechamente vigilados. Los objetivos de estos programas son los siguientes:

- Declarados

- Ahorrar costos con medidas alternativas que son más baratas que el uso de la prisión

- Prevenir la comisión de delitos por la generalidad

- Proteger a la comunidad mediante el ejercicio de más control que la que hace la supervisión tradicional

- Rehabilitar a los ofensores mediante el uso de requerimientos obligatorios y la revocación de las órdenes violadas.

- No declarados

- Crear una apariencia de reforma correccional

5 Anne Worrall \& Clare Hoy. Punishment in the Community. Managing the offenders, making choices. Portland: Willand Publishing House, 2005 (1 ${ }^{\text {a }}$ edición 1977). 
- Encontrar una forma de reclamar los recursos limitados para las sanciones que no suponen privación de la libertad

- Permitir que la administración y los políticos respondan al sentimiento punitivo de la ciudadanía.

Las primeras medidas no privativas de la libertad fueron las sanciones pecuniarias, que estaban previstas para conductas que no revestían suficiente gravedad como para ameritar la privación de la libertad, pero que sí se consideraba que merecían algún tipo de sanción penal. Estas medidas fueron objeto de críticas, pues el incumplimiento de la multa estaba asociado a la pena privativa de la libertad, de manera que quien no la pagaba era objeto de privación de su libertad. ${ }^{6}$ Pero otras sanciones se fueron desarrollando en el siglo XIX y XX y se clasificaban como sanciones auto - regulatorias, basadas en el uso de la vergüenza (shaming penalties); sanciones financieras, como la multa, a la que se le critica positivamente porque no supone un estigma para el infractor, pero se le critica que tampoco supone una rendición de cuentas por parte del mismo; sanciones supervisadas, pues se asume que el infractor carece de la fuerza de voluntad suficiente para reparar los lazos rotos con la comunidad y, por ello, requiere un impulso por parte de un supervisor. Dentro de este tipo de sanciones se encuentran las ordenes de rehabilitación dentro de la comunidad; las ordenes de supervisión; las órdenes de restricción de movimientos; las ordenes de atender un centro determinado; las sanciones que suponen un trabajo en beneficio de la comunidad; las ordenes de reparación para los menores de 17 años; y las ordenes de tratamiento de drogas y de exámenes continuos de consumo.

Otro tipo de sanciones son el binding over $^{7}$ pero requiere una garantía pecuniaria si el compromiso verbal del ofensor es violado; una sentencia suspendida, que ofrece un periodo de auto-regulación para el infractor, pero requiere que vuelva ante el juez y sin que exista garantía que éste considere que la sanción penal no es merecida. Esta medida es criticada pues la centralidad

6 Un régimen tal existía bajo el Código Penal de 1980.

7 Es una medida que impone obligaciones a la persona y la somete a ciertas condiciones, algunas de ellas financieras. 
de la prisión aquí es evidente, pues el infractor sabe que solo ha escapado a la prisión porque ha cumplido con las condiciones, y no porque realmente haya auto-regulado su conducta. Sin embargo, como se verá al analizar los TTD, la motivación que ofrece evitar el uso de la prisión no puede ser sobrevalorada.

En contra de estos mecanismos de sanción comunitaria se dice que son muy suaves contra el delito, que amplían la red de control del sistema, ${ }^{8}$ y que tiene un amplio potencial de discriminar a las personas por razón de su sexo, raza o clase (Worral \& Hoy, 2005: 22).

Las estrategias varían en sus intenciones: algunas buscan prevenir la comisión de nuevos delitos, otras los efectos del sistema penal, otras reducir el uso del sistema penal en casos que no se consideran tan graves pero que no ameritan la legalización. En todo caso, algunas de estas medidas apuntan a reducir los efectos negativos sobre las personas y con ello afectar su comportamiento hacia el futuro, de modo que se logre la protección de la ciudadanía al tiempo que se presta ayuda a los infractores. Se trata de medidas que buscan una adecuada combinación entre seguridad de la ciudadanía e inclusión social de los infractores. Algunos de los programas que se presentan como parte de las sanciones en la comunidad son los siguientes:

- Money Payment Supervisión Orders MPSOs que buscan garantizar la situación financiera del infractor y le obligan a solucionarla en conjunto con el oficial de libertad controlada (Probation).

- Tratamiento de drogas y órdenes de pruebas continúas. Estos programas fueron introducidos en el Reino Unido en el año 1998 y estuvieron disponibles para las cortes a partir del año 2000. Suponía la imposición de una sentencia leve, pero con un control mucho más estricto de su cumplimiento. El propósito no es acabar con el consumo como tal, sino simplemente eliminar la relación entre consumo y comportamiento criminal (esto es, la comisión de

8 Stanley Cohen. Visions of Social Control. Crime, punishment and classification. Cambridge: Polity, 1985; Massimo Pavarini. Castigar al enemigo: Criminalidad, exclusión e inseguridad. Quito: Flacso, 2009. 
delitos para el sostenimiento de su adicción). Los estudios sobre la efectividad de estos programas muestran que la tasa de reincidencia entre quienes ingresan a ellos es más baja que en quienes no terminan el programa, por lo que aconsejan estimular la culminación del mismo y desarrollar medidas para evitar el abandono.

- Police Offenders projects. Estos son programas desarrollados por la policía, que considera que la mayor parte de las infracciones son cometidas por los mismos infractores, por lo que recomiendan concentrar la acción policiva en estas personas. Sin embargo, no se trata simplemente de la labor de la policía, sino que combina su trabajo con el de otras agencias, de manera que diversos aspectos de la vida de los infractores sean tenidos en cuenta (Worrall \& Hoy, 2005: 158). La justificación de estas medidas es que si una de ellas funciona, se debe implementar, de manera que cada vez más se amplíe la gama de medidas que funcionan en la prevención del delito y en la reducción del número de personas privadas de la libertad. ${ }^{9}$

- Personal Support for Developing Learning Pathways in Custody PSIC pilot Project. Este programa se creó en Gales con el fin de ayudar a los jóvenes que se encontraban en situación de exclusión. El proyecto coincide con otros programas más ortodoxos en la búsqueda de acceso a educación y entrenamiento y en ayudar a los jóvenes infractores en hacer su tránsito de la situación de custodia a la de vida en la comunidad. ${ }^{10}$ Este programa muestra que el apoyo a los jóvenes en ayudarlos a desarrollar habilidades sociales y emocionales es importante para sostener el deseo al cambio. Simplemente adoptar una política de ser duros con el delito no funciona, y se requieren programas que garanticen el logro de los objetivos de reducción del delito. La estrategia busca tratar a los ofensores primero como

9 Jo Brayford, Francis Cowe, John Deering. What else Works. Creative work with offenders. Portland: Willand Publishing, 2010.

10 Jo Brayford \& Duncan Holtom. "Dancing through gaps: a Welsh approach to personal support in custody for Young people”. En Brayford et al. 2010. Pp. 169 - 187. 
niños o adolescentes y en segundo lugar como ofensores. Esto es, se reconoce que la causa del delito tiene que ver con la infancia o la adolescencia y con la exclusión social, y se apunta a la solución de esos problemas con independencia de la comisión del delito. En todo caso, son medidas post penitenciarias por lo que debe tenerse en cuenta que ya se ha dado un proceso de exclusión significativo. ${ }^{11}$ El programa se vale de los cinco dominios para ayudar a los jóvenes a desarrollar sus capacidades: auto consciencia; manejo del humor; empatía; manejo de las relaciones sociales; auto motivación (Brayford \& Holtom, 2010: 181). La evidencia empírica muestra que los jóvenes no solo necesitan algo más que la pena para cambiar, ellos necesitan la motivación para hacerlo. Requieren la habilidad y la capacidad para el cambio, y esto es lo que el programa busca darles. El apoyo no proviene de otros adultos sino de otros jóvenes voluntarios, con quienes trabajan la motivación para el cambio. $\mathrm{Si}$ bien se reconoce que es un programa costoso, también se recuerda que la medida tradicional, la prisión, es más costosa y ha demostrado no cumplir el objetivo de prevenir el delito y la reincidencia.

- En los Estados Unidos recientemente se anunció la creación del grupo Law Enforcement Leaders to Reduce Crime and Incarceration con el fin de unir fuerzas entre los fiscales locales, estatales y federales con el fin de reducir tanto el delito como la población carcelaria. El grupo, que cuenta con el apoyo de la policía, afirma que no se debe enviar a todas las personas a la cárcel, solo aquellas que realizan la mayoría de actos violentos. Para el grupo, hay una diferencia entre poner un arma en la cara de un persona y ser capturado con dos bolsas de heroína porque se tiene un problema. Se trata de dos delitos diferentes, y las prioridades de la política criminal deben estar reflejadas en la ley.

$11 \mathrm{La}$ experiencia catalana en el manejo de las pandillas latinoamericanas es muy similar. En lugar de tener en cuenta su característica de infractores ha tenido en cuenta su característica como migrantes y miembros de la pandilla. Se reconocen los lazos familiares de la pandilla y el hecho que sirve como medio para paliar la soledad de la migración. 
El grupo busca que se apruebe una reforma a la legislación penal que incluya la reducción de algunos mínimos mandatorios por delitos relacionados con drogas y disminuir el uso del confinamiento solitario para jóvenes en las prisiones federales. ${ }^{12}$

- Back on Track. El programa fue establecido a partir del mes de septiembre de 1998 para ser aplicado a personas condenadas por conducir ebrias y que tengan más de una condena por este tipo de conductas. El programa tiene un costo que es asumido por los infractores. Esto con el fin de evitar la suspensión de la licencia de conducción.

Los requisitos para aprobar el programa son:

- No usar alcohol o drogas dentro de las 24 horas o en cualquier día en que se participe en el programa. Los medicamentos prescritos se pueden usar en tanto no impidan tomar parte en las actividades.

- Atender todas las sesiones y llegar a tiempo a ellas. En caso de no poder hacerlo avisar con 24 horas de anticipación. Sólo se permite faltar en ciertos casos y en todo caso se debe realizar la sesión en otro momento.

- Se hace una valoración y se determina si se prefiere el programa educativo o el de tratamiento.

- El taller de educación tiene como finalidad que los participantes aprendan sobre lo siguiente:

- Mitos y hechos acerca del alcohol y otras drogas

- Cómo el alcohol y otras drogas afectan la capacidad para conducir

- Las consecuencias legales de conducir ebrio o bajo la influencia de las drogas

- Las formas de evitar conducir en esta condición

- El taller dura un día (8 horas)

12 En http://www.huffingtonpost.com/entry/prisoner-crowding 56279b6ae4b08589ef49e13a Última visita 21 de octubre de 2015. 
- El taller sobre tratamiento incluye lo siguiente:

- Aprender por qué se bebe o usan otras drogas y cómo afecta nuestras vidas

- Aprender y tomar responsabilidad por el consumo de alcohol y otras drogas y sus consecuencias.

- Comprometerse a la superación del problema

- Aprender estrategias para manejar cosas como el estrés, la comunicación, la rabia y el ocio.

- Planes para evitar la recaída y tomar decisiones sanas

- Cómo evitar tomar y manejar al mismo tiempo

- El programa tiene sólo una duración de 2 días (16 horas). ${ }^{13}$

- Los Tribunales de Tratamiento de Drogas se ven como parte de este tipo de medidas de sanción en la comunidad. Así se presentan en la versión en español de un documento elaborado por el Departamento de Justicia sobre las diez características de los TTD:

Los tribunales de drogas se implementaron a fines de la década del ochenta pero no evolucionaron por sí solos, sino que fueron el resultado de la evolución constante de distintos enfoques basados en la comunidad y el trabajo en equipo. Estos enfoques derivan de programas innovadores desarrollados tanto por los organismos encargados de los servicios previos al juicio y de la libertad condicional, como por las asociaciones que se centran en el tratamiento del acusado, por ejemplo, Tratamientos Alternativos para una Comunidad Segura (TASC, por sus siglas en inglés), y también de las innovaciones en el marco del cumplimiento de la ley, como los programas de vigilancia policial comunitaria.

Sin embargo, los tribunales de drogas no han sido la culminación

13 En http://www.remedial.net/about.html Última visita 21 de octubre de 2015. 
ni el punto central de la evolución en los programas judiciales basados en la comunidad. En los últimos años, el éxito obtenido por los tribunales de drogas ha alentado el crecimiento de los "tribunales comunitarios" y hoy incluyen tribunales para la violencia doméstica, para el control de alcoholemia durante la conducción de un vehículo, para el consumo de drogas en menores y en familias, para problemas vecinales e incluso para los casos en que los padres eligen no mantener a sus hijos (que se conocen como deadbeat dad en los EE.UU.). Estos tribunales están diseñados para reflejar las preocupaciones y prioridades de las comunidades, acceder a sus recursos, incluir a las organizaciones comunitarias en las decisiones que tomen los funcionarios y lograr el apoyo y la participación general.

Los tribunales de drogas, junto con los demás programas judiciales basados en la comunidad que conformen el campo judicial de una comunidad, se consideran parte del campo de la "justicia comunitaria". Estos programas al igual que los de vigilancia policial, acciones judiciales y sistemas penitenciarios comunitarios están evolucionando rápidamente, y con mucho ímpetu, logrando expandirse por todos los Estados Unidos. El siglo XXI seguirá siendo testigo del progreso del campo de la justicia comunitaria y, por ende, de los tribunales de drogas.

\section{Justicia Restaurativa}

La filosofía que fundamenta las diferentes alternativas a la pena privativa de la libertad es la de la justicia restaurativa, pues no se trata simplemente de abaratar los costos del sistema de justicia penal o de reducir el número de personas en prisión, sino de reparar los lazos y las relaciones rotas con la comisión del delito. La justicia restaurativa se ha definido como aquellos procesos que buscan 
involucrar a las víctimas, los infractores y algunos miembros de la comunidad en deliberaciones sobre el impacto de una infracción particular y las formas más apropiadas de responder a ella. Las medidas que simplemente buscan compensar a las víctimas, prestar un servicio a la comunidad o crear consciencia sobre las víctimas no pueden ser consideradas medidas restaurativas porque no incluyen a todos lo protagonistas en el proceso de decisión. Pero entre los que sí son incluyentes están la mediación entre las víctimas y los infractores; las diferentes formas de conversación entre víctimas e infractores; y los paneles de ciudadanos (Dignan, 2007: 269). Este tipo de medidas se pueden dividir de la siguiente manera (Dignan, 2007):

- Medidas alternativas a la investigación de la fiscalía

o En algunos países la Policía cuenta con poderes discrecionales para pasar los casos al sistema penal. En estos casos, los infractores fueron objeto de cauciones y fueron desviados hacia sistemas de mediación y de reparaciones.

o En la Policía del valle del Támesis se desarrolló un sistema que se caracterizaba por una aproximación predeterminada por parte de la policía, en la que ésta estimulaba al infractor a reconocer el daño causado con su delito. Esta conversación se conoce como "caución restaurativa", en donde solo toman parte el facilitador, el infractor y los miembros de la familiar del infractor. Si se trata de una "conferencia restaurativa" está presente la víctima; y si es una "conferencia comunitaria" están también presentes miembros de la comunidad. Estas medidas han recibido apoyo de las policías locales, pero no es una medida ampliamente extendida en el Reino Unido. En el caso de los programas con jóvenes, se busca mostrarles el impacto de su acto en las personas y que tomen responsabilidad por lo que han hecho. La medida busca que entiendan el impacto que un delito tiene en su vida futura. 
o En varios países de la Unión Europea se permite a la policía derivar el caso a instancias no penales, cuando ello es posible y cuando no hay necesidad de judicializarlo. Esto puede consistir en regaños a adolescentes hasta acuerdos entre la víctima y el victimario promovidos por la policía. En todo caso la Unión Europea sugiere que las Fiscalías den instrucciones claras a los miembros de la policía para que se use este mecanismo de manera correcta. ${ }^{14}$

- Medidas sustitutas del proceso convencional de imposición de una sentencia

o Los youth offender panel parten de la base del postulado básico de que la justicia restaurativa no puede determinar la responsabilidad de una persona. Un joven infractor debe reconocer su responsabilidad y en cambio es objeto de una orden de reenvío hacia el panel, compuesto por dos personas de la comunidad y un miembro del equipo local de jóvenes infractores, una organización multi - agencial que presta servicios a los jóvenes en conflicto con la ley. En este panel se discute con el joven y su familia el impacto de su conducta y se llega a un acuerdo que alcanza la forma de un contrato, de manera que la víctima y la familia del infractor forman también parte del acuerdo. Si el joven cumple con su parte del acuerdo, la condena impuesta se considera cumplida.

- Medidas que van con el proceso de imposición de una pena

o Aplazamiento de la condena. En ocasiones se impone no solo la sentencia, sino que en ella se imponen también, como acompañantes, algunas medidas restaurativas. Una de tales medidas es el aplazamiento de la condena, en el que el juez se abstiene de imponer pena alguna, hasta tanto el infractor

14 Matt Loffmann and Faye Morten. Investigating alternatives to imprisonment within Council of Europe member states. Brussels: The Quaker Council for European Affairs: 2010. 
no cumpla con ciertos requisitos y si los considera cumplidos, puede abstenerse al final de imponer pena alguna. Esto permite analizar el comportamiento de los infractores por un tiempo antes de imponer sentencia alguna y siempre sobre la base de los informes presentados por el agente de la libertad controlada (probation officer).

o Multas y ordenes de compensación. Se imponen sobre todo cuando se trata de un delito contra el patrimonio económico, y cuando el daño causado puede ser reducido a cuestiones meramente monetarias sin causar en la víctima o en el público la sensación de ausencia de justicia.

o Monitoreo electrónico. Esta ha mostrado ser una medida muy efectiva, pues permite la vida en libertad y se ha demostrado que la tasa de reincidencia es muy baja ( $8 \%)$.

o Sentencias suspendidas. Se suspende la sentencia a cambio del cumplimiento de ciertas condiciones y permite evitar el uso de la prisión, pues la persona aún no ha sido sentenciada. El cumplimiento de la sentencia se logra con la amenaza latente de que al momento de imponer la sentencia se haga uso de la pena privativa de la libertad.

o Sanciones y medidas aplicadas en la comunidad. Usualmente se trata de trabajo en beneficio de la comunidad, lo que permite a su vez que la persona se acerque a la comunidad afectada y que no se dé un proceso de exclusión social.

o Obligaciones de recibir tratamiento para superar el consumo problemático de alcohol o de drogas.

o Rehabilitación del autor de delitos sexuales. 
- Medidas que se imponen con posterioridad a la sentencia

o En estos casos se busca facilitar el regreso de la persona a la vida en libertad y reparar el daño causado con el delito. En Bélgica es el único lugar de Europa en donde se promueve sobre bases regulares medidas de justicia restaurativa con infractores que están privados de la libertad y en la etapa de cumplimiento de la sentencia. En otros países como Inglaterra, Gales y Escocia, las medidas son limitadas, ad-hoc y no cuentan con el apoyo del sistema de justicia penal.

o Probation. La libertad condicional es una de las medidas más comunes en los sistemas penales. Permite la liberación de la persona al cabo de cierto tiempo en prisión y a cambio impone ciertas condiciones luego de cuyo cumplimiento se otorga la libertad. Una de las críticas es que los oficiales de libertad condicional son muy estrictos frente a las violaciones y toman con mucha frecuencia la opción de enviar a la persona a prisión.

o Liberación anticipada. Se busca preparar a la persona para la vida en libertad y busca asistir a los presos en su retorno a la vida en libertad. Es importante que se involucre a la comunidad a la cual llega el infractor, pues de ellos depende que no se den las condiciones para una recaída en el delito.

\section{Fuentes de consulta}

Anthony Bottoms, Sue Rex \& Gwen Robinson. Alternatives to Prison. Options for an insecure society. Portland: Willan Publishing, 2004 Mark Kleiman. When brute force fails. How to have less crime and less punishment. Princeton: Princeton University Press, 2009.

R.A. Duff. "Penance, punishment, and the limits of the community". En Michael Tonry. Why punish? How much? A reader on punishment. 


\begin{abstract}
Oxford: Oxford University Press, 2011.
Anne Worrall \& Clare Hoy. Punishment in the Community. Managing the offenders, making choices. Portland: Willand Publishing House, 2005 ( $1^{\text {a }}$ edición 1977).

Stanley Cohen. Visions of Social Control. Crime, punishment and classification. Cambridge: Polity, 1985; Massimo Pavarini. Castigar al enemigo: Criminalidad, exclusión e inseguridad. Quito: Flacso, 2009.

Jo Brayford, Francis Cowe, John Deering. What else Works. Creative work with offenders. Portland: Willand Publishing, 2010.

Jo Brayford \& Duncan Holtom. "Dancing through gaps: a Welsh approach to personal support in custody for Young people". En Brayford et al. 2010.

Matt Loffmann and Faye Morten. Investigating alternatives to imprisonment within Council of Europe member states. Brussels: The Quaker Council for European Affairs: 2010.

\section{Fuentes de consulta electrónica}

http://www.huffingtonpost.com/entry/prisoner-crowding 56279b6ae4b0 8589ef49e13a

http://www.remedial.net/about.html
\end{abstract}

